\title{
Perinatal factors affecting the gut microbiota - are they preventable?
}

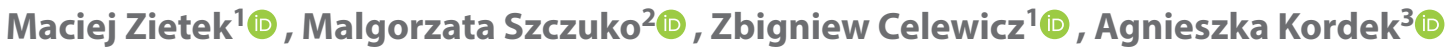 \\ ${ }^{1}$ Department of Perinatology, Obstetrics and Gynecology, Pomeranian Medical University, Szczecin, Poland \\ ${ }^{2}$ Department of Human Nutrition and Metabolomics, Szczecin, Poland \\ ${ }^{3}$ Department of Neonatology, Medical University in Szczecin, Poland
}

\begin{abstract}
Intestinal microbiota affects many aspects of physiological processes. The type of microbiota in the early stages of life is a critical element conditioning the development of the immune response and food tolerance. Disturbed colonization of the digestive tract resulting from the amount or diversity of bacteria colonies stimulates an inflammatory response that is associated in later life with inflammatory and autoimmune diseases. One of the elements disturbing normal colonization in the perinatal period is the operative way of delivery by caesarean section and the administration of antibiotics, used as a prophylactic measure as well as for therapeutic reasons. Based on the current state of knowledge, there is a lot of evidence demonstrating the long-term adverse effects of these modifying agents for gut microbiota, which should be kept to a minimum as far as possible.
\end{abstract}

Key words: gut microbiota; neonate; antibiotics; cesarean section

\section{INTRODUCTION}

In recent years, increasing attention has been focused on disorders of newborn gut colonization and their impact on its further development and functioning in adulthood, however, these data are largely not systematized. Initial microbial colonization is one of the most important factors affecting a child's development having a significant impact on triggering of diseases in his adult life. Such disorders as diabetes, obesity, allergies, inflammatory bowel diseases and other autoimmune disturbances are found to be linked with abnormal colonization during the first two years of life, that commence since the perinatal period. Epigenetic changes may induce transgenerational issues with fixation of mutant genes, which functional products have effect in subsequent generations. The perinatal factors that impair proper newborn's gut colonization include caesarean section, use of antibiotics during pregnancy and delivery, among others, as a prevention of GBS infection, as well as early abandonment of breastfeeding replaced by formula feeding. A recent significant increase in both the incidence of surgical deliveries as well as use of antibiotic therapy have become the reason for more and more frequent researches on the negative impact of these procedures.

\section{THE COLONIZATION OF THE FETUS IN UTERO}

The degree of sterility of the fetal environment and the possibility of transfer of the microbiome to the uterine cavity have been the subject of scientific discussion for a long time. For many years, it was believed that the fetal amniotic environment would remain sterile until the premature rupture of membranes had place during the delivery and that the intestinal tract of the newborn remain sterile until birth. According to the above paradigm, the first contact of a child with bacteria occurs through the vertical (from the mother) and horizontal (from the environment) way, and the first signs of bacterial colonization can be recognized only a few hours after delivery [1]. The implementing and use of highly specialized methods for microbial identification, including polymerase chain reaction (PCR), undermined the theory mentioned above and allowed for precise determination of the microbiota of the fetal environment [2-4]. A modified PCR technique, i.e. PCR-DGGE (polymerase chain reaction - denaturing gradient gel electrophoresis) is used 
to assess bacterial DNA and the 16S rRNA sequence in the material being examined (e.g. stool, placenta, amniotic fluid, meconium). The presence of bacteria in placental tissue, umbilical cord and meconium was confirmed, proving the physiological contact of the fetus with microbiota [2]. In several investigations it is reported that intrauterine colonization of the fetus plays a pivotal role in the maturation of the immune system and has a significant impact on its development in the neonatal, childhood and ultimately in adulthood [5]. Sodeborg et al. [6] showed a relationship between disturbed bacterial colonization in pregnant women complicated by obesity and diabetes, with excessive birth weight of their newborns, abnormal fat distribution, overweight, immune-related dysfunction and the presence of non-alcoholic fatty liver in their adult life. Studies confirming the association between abnormal intestinal colonization and autism in children have been also published [7]. If the "hypothesis of in utero colonization" would prove to be correct, the role of the primary microbiome, environment, lifestyle and clinical factors determining human health as well as regulatory epigenetic aspects should be redefined [8]. As consequence of this assumption the clinical activities promoting proper colonization with correct transfer of microbiota in pregnant women with among others reducing the incidence of cesarean sections should be implemented [1]. Human microbiota shows an individual variability, determined by the number and diversity of bacterial strains. During pregnancy, microbes and metabolites of the microbiome are transferred to the fetus and then to the newborn during labor and lactation [9]. The composition of the microbiome is moderated by the environment, including diet, pregnancy health, metabolic diseases, gestational weight gain, termination of pregnancy, genetic factors and antibiotics used in the perinatal period [10-12].

The native intestinal microflora differs in women with normal body weight and obesity. Pregnant women with excessive weight gain have shown an increase in Firmicutes (Staphylococcus) and some Proteobacteria (Escherichia coli), especially in the second half of pregnancy $[12,13]$. It has been shown that differences in microbiota species in pregnancy determine the increased birth weight of newborns $[10,12]$. One of the factors affecting the developing fetus in obese women is the excessive amount of plasma endotoxins, the complex lipopolysaccharides (LPS) forming an inherent fraction of the outer cell wall of bacteria. Endotoxins may increase the intestinal translocation of bacterial products across the intestinal mucosa, contributing to the formation of an inflammatory response within the placenta and insulin resistance. Collado et al. [2], using the $16 \mathrm{~S}$ rRNA sequencing method to identify bacterial species in amniotic fluid and placental tissue samples, found that the most common strain was the bacteria belonging to the Pro- teobacteria group, especially from the Enterobacteriaceae family (genera Escherichia and Shigella). The presence of the same microorganisms has been also identified in fetal vernix caseosa, meconium and faeces of newborns, although their growth was less abundant. Propionibacterium, in turn, was the second most common strain that has been found in placental tissue, amniotic fluid and meconium as well. Other bacterial strains have been also shown to be present, although in a much smaller quantity (Streptococcus, Staphylococcus, Lactobacillus) $[3,11]$.

\section{EARLY BACTERIAL COLONIZATION OF THE NEWBORN AFTER BIRTH}

According to Langherdries et al. [11], the postnatal contact of a newborn baby with an invasive microbiological environment and exposure to the new antigens, intestinal bacteria and their products, triggers immune mechanism within the intestinal tissues. It is likely that the modification of the quality of the host bacteria and their interaction leads to disturbed colonization, favoring the acquisition of abnormal immunity of neonates and an appearance of autoimmune and allergic diseases in later life. Various environmental factors occurring in the early neonatal period may affect the intestinal bacterial composition, potentially affecting the subsequent risk of diseases such as asthma, metabolic disorders and inflammatory bowel diseases [14]. The intestinal microbiome and adaptive immunity of infant is controlled by innate lymphoid cells (ILCs), which play an important role in coordination the inflammation, immunity, wound healing, and tissue homeostasis [15]. The above observations particularly apply to developing countries. Therefore, it should be recognized that the composition of the intestinal flora determines the type of immune response and the change in the profile of the intestinal flora, the so-called dysbiosis precedes the development of allergies in later life of infants. The mechanism responsible for the regulatory effect of a microbiota on the body is the ability to change gene expression in the host gut via the gut flora. Bacteria mainly colonize the large bowel, forming colonies containing an average of 1011 CFU (Colony Forming Unit) in 1 gram of tissue tested. Their content during life does not change much in healthy individuals, provided that there are no infectious diseases and antibiotic therapy. The anaerobic microflora as the most important is represented in $80 \%$ by strains of the genera: Bacteroides, Eubacterium, Bifidobacterium, Peptostreptococcus. The factor initiating colonization of the intestine is a direct contact with maternal rectovaginal flora during delivery, followed by breastfeeding $[1,4,16]$. Usually this process lasts several weeks. During the first 48 hours, the number of bacterial cells is already very high, 104-106 CFU/mL of intestinal content, and is independent of how the neonate is fed. During this period, antibiotic therapy is a factor that 
significantly disturbs the quality and quantity of colonizing bacteria. Over the next 10 days, an increase in Bifidobacteria and Lactobacillus bacteria number is observed, achieving a stable concentration of approximately $109 \mathrm{CFU} / \mathrm{mL}$ of intestinal content. In the next phase of colonization, an increase in Escherichia coli, Bacteroides spp and a much less significant increase in Clostridiae are detected. In nonphysiological conditions during the perinatal period, it is the Clostridia strains that show the strongest positive association with increased neonatal morbidity $[11,12,17]$. At the end of the first month of life, there are differences in the type of bacterial colony, depending on how the newborn is fed. In the breast-fed babies' group, the intensive intestinal growth relates to the Bifidobacteria colony, compared with only $30-40 \%$ bacterial content in formula-fed babies. By the end of the first and up to the second year of life, the content of intestinal microflora is approaching that in adulthood.

\section{THE GUT MUCOSAL IMMUNE SYSTEM}

A particularly important element of acquired immunity is the intestinal mucosa barrier. Commensal intestinal microflora determines the functions of lymph tissue, occurring within the gastrointestinal tract, the so-called GALT (gut-associated lymphoid tissue) by transmitting information to enterocytes and $M$ cells of the intestinal lymphoid epithelium. The GALT system fulfills a dual role: it induces an immune system against bacterial pathogens and viruses through an enhanced, well-controlled pro-inflammatory response and increases complex immune mechanisms that produce antigen tolerance [12]. The initial colonization of gut microorganisms depends on the GALT maturation and is regulated by the Gastrointestinal-Blood Barrier (GIB) throughout selective absorption to the blood as well. The establishment of a balance between immunity and infections is determined by GALT and critical closure of GIB. Enterocytes, belonging to the major histocompatibility complex (MHC), can partially transfer information about the type of antigen, thus fulfilling the role of the first mucosal immunity mechanism. The interaction of enterocytes with endothelial T lymphocytes, mainly CD8 + (cytotoxic T lymphocytes) is of fundamental importance here. The degree of neonatal intestinal maturity varies individually and shows differences depending on the week of gestation [18]. Although the human intestine reaches structural maturity around 19 weeks of pregnancy and all cellular elements of the intestinal immune system are already present, its functional full maturity occurs after the onset of antigenic stimulation with colonizing microflora [18]. Studies have shown that premature exposure of the fetal intestine to microorganisms contained in the amniotic fluid may be associated with premature birth [19]. Also, the exposure of a pregnant woman's body to bacteria may affect the development and maturation of the fetal epithelium of the intestine, which in turn promotes its hypersensitivity to pro-inflammatory factors, leading, among others to necrotic enterocolitis in a newborn [20]. Newborns during labor are exposed to maternal bacteria of the vagina and anus mucosa, which are mainly facultative anaerobes, Streptococci [18]. Within a few days, Enterococci and enterobactriaceaea appear, whose activity is associated with a decrease in oxygen concentration, thus facilitating further colonization with Bifidobacteria and Bacteroides spp. and Clostridium spp. Exposure of the nascent baby to ligands of colonizing bacteria seems to be a key factor developing an intestinal tolerance. Bacterial ligands are recognized by innate immune receptors, Toll like Receptors (TLRs), whose expression is present in fetal, neonatal and adult life. TLR2 and TLR4 are present in the fetal intestinal epithelium since the $18^{\text {th }}$ week of gestation [21]. As a result of receptors activation that occurs throughout the contact of newborn's intestinal epithelial cells with LPS, an endotoxin present in the outer membrane of Gram-negative bacteria, the immunotolerance process develops [22]. Perinatal contact of bacterial LPS with fetal epithelial TLR induces the production of microRNA (miR) -146a, which in turn inhibits the translation of interleukin-1 receptor-associated kinase (IRAK-1), a key enzyme in the TLR4 metabolic pathway. Low IRAK-1 concentration protects the intestine during the first contact with bacteria. After recognition of microorganisms by a newborn's intestinal epithelium, it also begins to produce cytokines such as interleukin-10 (IL-10) and transforming growth factor beta (TGF- $\beta$ ), which have immunoregulatory properties. Among the secreted molecules responsible for determining microbiological tolerance homeostasis, the peptides defensin (alpha and beta) and cathelicidin play an important role. In other words, there are antimicrobial peptides that regulate commensal flora and protect against pathogens. Located in the small intestinal crypts, Paneth cells produce large amounts of alpha defensin, an antimicrobial peptide which accounts for over $70 \%$ of their secretory bactericidal activity. In turn, beta defensins are mainly present in the large intestine. In contrast, cathelicidins are peptides expressed in the intestinal epithelium and produced by various intestinal cells, including neutrophils, mast cells, and epithelial cells. The bactericidal activity of these cationic peptides is associated with their amphipathic properties. Endogenous cathelicidin affects the intestinal barrier integrity and modulates the infiltration of neutrophils and macrophages during infection and bacterial sepsis [23].

\section{MODE OF DELIVERY}

One of the factors disturbing the proper colonization of the newborn's intestine is an operative delivery by caesarean section [17,24]. Differences in newborns' gut microbiome content and diversity on the $4^{\text {th }}$ and $120^{\text {th }}$ day after delivery 
have been shown, suggesting that the composition of the so-called early postpartum microbiota determines the microbiota during the subsequent years. The changes in gut bacteria populations diversity, however, largely disappear after six months of life of the infant. [24]. In case of caesarean section delivery, colonization of the newborn's intestine seems to occur later in relation to infants born by vaginal route. Many studies have also shown that microbiota of newborns after caesarean section differs from that of delivered by vaginal route $[25,26]$. In an animal model, it was demonstrated that the initial bacterial flora of newborns is mainly composed of microorganisms of the rectovaginal origin of mothers [18]. In the case of neonates after caesarean section, the dominant colonizing microorganisms are Staphylococcus and Propionibacterium, derived from the mother's skin. On the other hand, vaginal birth is associated with colonization mainly by Lactobacillus and Prevotella strains occurring in the female urogenital area [3]. The most efficient colonization of a baby, therefore, occurs along with its inoculation with vaginal discharge microbiota during childbirth. During childbirth, the baby is also colonized by maternal digestive tract bacteria [27]. It is likely, that operative way delivery seems to be associated with a higher incidence of asthma, inflammatory bowel disease and obesity in adulthood through disturbed colonization of the neonate leading to dysbiosis [2]. Other factors affecting the development of a newborn microbiota are also the duration of pregnancy, diet during pregnancy, breastfeeding, genetic and environmental conditions.

\section{PERINATAL ANTIBIOTIC THERAPY}

Exposure to antibiotics is associated with their destructive effects on the intestinal bacterial flora. The implementation of both early short-term and prolonged antibiotic therapy may be associated with an increased risk of being overweight or obese in late childhood as well as other fat accumulation related disturbances [28]. The use of antibiotic therapy during labor in women GBS positive pregnant women as a prevention of infectious complications in newborns, including sepsis, significantly disturbs the microbial balance of the rectovaginal region, impairing the proper colonization of the newborn during delivery [29]. Also, pregnancy exposure to antibiotic therapy used in both premature babies and full-term children significantly affects the composition of the microbiota of newborns, increasing the risk of early neonatal sepsis [29]. The effect of perinatal antibiotic therapy inducing maternal vaginal and infant gut microbiota dysbiosis, is a significant decrease in the number of Lactobacillus spp. In Fouhy F. et al. [30] study, carried out in nine full-term newborns treated with ampicillin and gentamicin, the exposure to antibiotics was associated with an increase in growth of Proteobacteria colony and decrease of Actinobacteria in faeces, especially of Bifidobacterium species within four weeks, compared to unexposed for antibiotics newborns. According to Zhou et al. [29], the neonates born at term without antibiotic exposure showed no early sepsis, while in the group of neonates born at term or prematurely exposed to antibiotic therapy before labor, early-onset sepsis has been diagnosed in at least one case. Therefore, antibiotics routinely used in the perinatal period may cause gut dysbiosis, resulting in both short- and long-term diseases. Early empirical antibiotics in newborns increase the risk of necrotizing enterocolitis (NEC) and late-onset sepsis (LOS) in premature babies, while maternal intrapartum antibiotic prophylaxis (IAP) is associated with inflammatory bowel diseases, obesity and atopic diseases in infants [31]. Antibiotics have a negative impact on the homeostasis of the pregnant woman's microbiota, while the use of properly selected probiotics has a beneficial effect on both pregnant women and their offspring. In order to prevent metabolic complications and in the case of therapeutic intervention, it is recommended to use good-quality pre- and probiotics in combination with an individually selected, high-nutritious diet [32]. The use of antibiotics during the first half of a child's life is associated with systematic excessive weight gain at a later time, while this effect is noticeable when women took medicine in the second and third trimester of pregnancy, as this correlation has not been seen in the first trimester of pregnancy. The consequences of taking antibiotics were still visible in children in the age of two years [33, 34]. The type and duration of antibiotic administration is important in the context of the effects on the pregnant women microbiota and the development of the child's immune tolerance. For this reason, according to the researchers, the use of antibiotics should remain under strict control, not only because of the possibility of the evolution of antibiotic-resistant microorganisms, but also because of the long-term metabolic consequences [28].

\section{SUMMARY}

According to the current state of knowledge, preliminary microbiotic colonization begins before the onset of labor and the microbiota derived from the amniotic fluid, placenta and maternal intestine may support its development in the infant. There are perinatal factors connected with a higher risk of impairing infants and children health, such as exposure to antibiotics and cesarean section. These two factors seem to be extremely important and may have a destructive impact on the infants' microbiota, leading to dysbiosios and subsequent health disorders. The presence of Lactobacillus and Bifidobacteria species reduces the risk of intestinal inflammation, while the Firmicutes/Bacteroides strains growth increases the risk of type 2 diabetes, overweight, obesity and lipid disorders, which is particularly visible in premature babies. The gut of infants born by cesarean section are mostly 
colonized by environmental skin bacteria with decreased diversity. Also, cesarean section results in delayed colonization, leading to weaken beneficial immunomodulatory signals.

It is extremely important for both women and physicians to be aware of the harmful use of antibiotic therapy in unjustified cases, as well as the use of cesarean section in conditions where there are no obstetric contraindications to terminate pregnancy by nature. The use of antibiotics should remain under strict control, not only because of the possibility of the evolution of antibiotic-resistant microorganisms, but also because of the long-term metabolic consequences. Considering the above facts may contribute to reducing the incidence of lifestyle diseases.

\section{Conflict of interest}

The authors declare no conflict of interest.

\section{Financial}

All authors have no financial relationships relevant to this article.

\section{REFERENCES}

1. Perez-Muñoz ME, Arrieta MC, Ramer-Tait AE, et al. A critical assessment of the "sterile womb" and "in utero colonization" hypotheses: implications for research on the pioneer infant microbiome. Microbiome. 2017; 5(1): 48, doi: 10.1186/s40168-017-0268-4, indexed in Pubmed: 28454555.

2. Collado MC, Rautava S, Aakko J, et al. Human gut colonisation may be initiated in utero by distinct microbial communities in the placenta and amniotic fluid. Sci Rep. 2016; 6: 23129, doi: 10.1038/srep23129, indexed in Pubmed: 27001291.

3. Walker RW, Clemente JC, Peter I, et al. The prenatal gut microbiome: are we colonized with bacteria in utero? Pediatr Obes. 2017; 12 Suppl 1:3-17, doi: 10.1111/ijpo.12217, indexed in Pubmed: 28447406.

4. Neu J. Developmental aspects of maternal-fetal, and infant gut microbiota and implications for long-term health. Matern Health Neonatol Perinatol. 2015; 1: 6, doi: 10.1186/s40748-015-0007-4, indexed in Pubmed: 27057323.

5. Ferretti P, Pasolli E, Tett A, et al. Mother-to-Infant Microbial Transmission from Different Body Sites Shapes the Developing Infant Gut Microbiome. Cell Host Microbe. 2018; 24(1): 133-145.e5, doi: 10.1016/j. chom.2018.06.005, indexed in Pubmed: 30001516.

6. Soderborg TK, Borengasser SJ, Barbour LA, et al. Microbial transmission from mothers with obesity or diabetes to infants: an innovative opportunity to interrupt a vicious cycle. Diabetologia. 2016; 59(5): 895-906, doi: 10.1007/s00125-016-3880-0, indexed in Pubmed: 26843076.

7. Li Q, Han Y, Dy AB, et al. The Gut Microbiota and Autism Spectrum Disorders. Front Cell Neurosci. 2017; 11: 120, doi: 10.3389/fncel.2017.00120, indexed in Pubmed: 28503135.

8. Kumar H, Lund R, Laiho A, et al. Gut microbiota as an epigenetic regulator: pilot study based on whole-genome methylation analysis. mBio. 2014; 5(6), doi: 10.1128/mBio.02113-14, indexed in Pubmed: 25516615.

9. Van Daele $E, \mathrm{Knol} J$, Belzer C. Microbial transmission from mother to child: improving infant intestinal microbiota development by identifying the obstacles. Crit Rev Microbiol. 2019; 45(5-6): 613-648, doi: 10.1080/1040841X.2019.1680601, indexed in Pubmed: 31859540.

10. Korpela K, Zijlmans MAC, Kuitunen M, et al. Childhood BMI in relation to microbiota in infancy and lifetime antibiotic use. Microbiome. 2017; 5(1): 26, doi: 10.1186/s40168-017-0245-y, indexed in Pubmed: 28253911.

11. Langhendries JP. [Early bacterial colonisation of the intestine: why it matters?]. Arch Pediatr. 2006; 13(12): 1526-1534, doi: 10.1016/j. arcped.2006.09.018, indexed in Pubmed: 17079124.

12. Houghteling PD, Walker WA. Why is initial bacterial colonization of the intestine important to infants' and children's health? J Pediatr Gastroenterol Nutr. 2015; 60(3): 294-307, doi: 10.1097/MPG.0000000000000597, indexed in Pubmed: 25313849.
13. Zhou $L$, Xiao X. The role of gut microbiota in the effects of maternal obesity during pregnancy on offspring metabolism. Biosci Rep. 2018; 38(2), doi: 10.1042/BSR20171234, indexed in Pubmed: 29208770.

14. Chong CY, Bloomfield FH, O'Sullivan JM. Factors Affecting Gastrointestinal Microbiome Development in Neonates. Nutrients. 2018; 10(3), doi: 10.3390/nu10030274, indexed in Pubmed: 29495552.

15. Yu JC, Khodadadi $H$, Malik A, et al. Innate Immunity of Neonates and Infants. Front Immunol. 2018; 9: 1759, doi: 10.3389/fimmu.2018.01759, indexed in Pubmed: 30105028.

16. Yang I, Corwin E, Brennan P, et al. The Infant Microbiome. Nursing Research. 2016; 65(1): 76-88, doi: 10.1097/nnr.0000000000000133.

17. Nagpal R, Tsuji $H$, Takahashi T, et al. Gut dysbiosis following C-section instigates higher colonisation of toxigenic Clostridium perfringens in infants. Benef Microbes. 2017; 8(3): 353-365, doi: 10.3920/BM2016.0216, indexed in Pubmed: 28504574.

18. Tourneur $E$, Chassin C. Neonatal immune adaptation of the gut and its role during infections. Clin Dev Immunol. 2013; 2013: 270301, doi: 10.1155/2013/270301, indexed in Pubmed: 23737810.

19. Peelen MJ, Luef BM, Lamont RF, et al. PREBIC Biomarker Working Group 2014-2018. The influence of the vaginal microbiota on preterm birth: A systematic review and recommendations for a minimum dataset for future research. Placenta. 2019; 79: 30-39, doi: 10.1016/j.placenta.2019.03.011, indexed in Pubmed: 31047708.

20. Baldassarre ME, Di Mauro A, Capozza M, et al. Dysbiosis and Prematurity: Is There a Role for Probiotics? Nutrients. 2019; 11(6), doi: 10.3390/nu11061273, indexed in Pubmed: 31195600.

21. Fusunyan RD, Nanthakumar NN, Baldeon ME, et al. Evidence for an innate immune response in the immature human intestine: toll-like receptors on fetal enterocytes. Pediatr Res. 2001; 49(4): 589-593, doi: 10.1203/00006450-200104000-00023, indexed in Pubmed: 11264445.

22. Stockinger S, Hornef MW, Chassin C. Establishment of intestinal homeostasis during the neonatal period. Cell Mol Life Sci. 2011; 68(22): 36993712, doi: 10.1007/s00018-011-0831-2, indexed in Pubmed: 21952827.

23. Ho J, Chan $\mathrm{H}$, Liang $\mathrm{Y}$, et al. Cathelicidin preserves intestinal barrier function in polymicrobial sepsis. Crit Care. 2020; 24(1): 47, doi: 10.1186/s13054-020-2754-5, indexed in Pubmed: 32041659.

24. Rutayisire $\mathrm{E}$, Huang $\mathrm{K}$, Liu $\mathrm{Y}$, et al. The mode of delivery affects the diversity and colonization pattern of the gut microbiota during the first year of infants' life: a systematic review. BMC Gastroenterol. 2016; 16(1): 86, doi: 10.1186/s12876-016-0498-0, indexed in Pubmed: 27475754.

25. Akagawa S, Tsuji S, Onuma C, et al. Effect of Delivery Mode and Nutrition on Gut Microbiota in Neonates. Ann Nutr Metab. 2019; 74(2): 132-139, doi: 10.1159/000496427, indexed in Pubmed: 30716730.

26. Liu Yu, Qin S, Song Y, et al. The Perturbation of Infant Gut Microbiota Caused by Cesarean Delivery Is Partially Restored by Exclusive Breastfeeding. Front Microbiol. 2019; 10: 598, doi: 10.3389/fmicb.2019.00598, indexed in Pubmed: 30972048.

27. Makino $\mathrm{H}$, Kushiro $\mathrm{A}$, Ishikawa $\mathrm{E}$, et al. Mother-to-infant transmission of intestinal bifidobacterial strains has an impact on the early development of vaginally delivered infant's microbiota. PLoS One. 2013; 8(11): e78331, doi: 10.1371/journal.pone.0078331, indexed in Pubmed: 24244304.

28. Turta O, Rautava S. Antibiotics, obesity and the link to microbes - what are we doing to our children? BMC Med. 2016; 14:57, doi: 10.1186/s12916016-0605-7, indexed in Pubmed: 27090219.

29. Zhou P, Zhou Y, Liu B, et al. Perinatal Antibiotic Exposure Affects the Transmission between Maternal and Neonatal Microbiota and Is Associated with Early-Onset Sepsis. mSphere. 2020; 5(1), doi: 10.1128/mSphere.00984-19, indexed in Pubmed: 32075882.

30. Fouhy $\mathrm{F}$, Guinane $\mathrm{CM}$, Hussey $\mathrm{S}$, et al. High-throughput sequencing reveals the incomplete, short-term recovery of infant gut microbiota following parenteral antibiotic treatment with ampicillin and gentamicin. Antimicrob Agents Chemother. 2012; 56(11): 5811-5820, doi: 10.1128/AAC.00789-12, indexed in Pubmed: 22948872.

31. Ficara $M$, Pietrella $E$, Spada $C$, et al. Changes of intestinal microbiota in early life. J Matern Fetal Neonatal Med. 2020; 33(6): 1036-1043, doi: 10.1080/14767058.2018.1506760, indexed in Pubmed: 30058404.

32. Lv Y, Yan Zi, Zhao $X$, et al. The effects of gut microbiota on metabolic outcomes in pregnant women and their offspring. Food Funct. 2018; 9(9): 4537-4547, doi: 10.1039/c8fo00601f, indexed in Pubmed: 30101246.

33. Mueller NT, Whyatt R, Hoepner L, et al. Prenatal exposure to antibiotics, cesarean section and risk of childhood obesity. Int J Obes (Lond). 2015; 39(4): 665-670, doi: 10.1038/ijo.2014.180, indexed in Pubmed: 25298276.

34. Cassidy-Bushrow AE, Burmeister $C$, Havstad S, et al. Prenatal antimicrobial use and early-childhood body mass index. Int J Obes (Lond). 2018; 42(1): 1-7, doi: 10.1038/ijo.2017.205, indexed in Pubmed: 28925412. 